\title{
Forthcoming Foot and Ankle Conferences 2015-16
}

American Academy of Orthopaedic Surgeons and the American Orthopaedic Foot and Ankle Society

Traumatic and Sports Injuries of the Foot and Ankle

February 26-28, 2014

Rosemont, Illinois, USA

www.aaos.org/3343

American Orthopaedic Foot and Ankle Society Specialty Day

March 28, 2015

Las Vegas, Nevada, USA

www.aaos.org

21st Congress of the Argentina Society of Medicine and Surgery of the Foot and Leg (SA, MeCiPP)

7th Congress of the Federation of Latin American Medicine and Surgery of the

Leg and Foot (FLAMECIPP)

April 14-18, 2015

Mar del Plata, Argentina

secretariasamecipp@yahoo.com.ar

17th European Foot and Ankle Society Instructional Course

April 23-24, 2015

Elzenveld, Antwerpen Belgium, in association with DOFAA and BFAS

http://register.eventplus.ie/

American Orthopaedic Foot and Ankle Society

Orthopaedic Foot and Ankle Surgery: The Essentials Course

April 23-25, 2015

Pittsburgh, Pennsylvania, USA

www.aofas.org

European Foot and Ankle Society Specialty Day at EFORT Congress

May 27-29, 2015

Prague, Czech Republic

http://register.eventplus.ie/

Indo Parekh 2015 Mid-Year Conference, Cidade De Goa, Goa, India

June 26-27, 2015

http://www.footandanklecourse.com/

American Orthopaedic Foot and Ankle Society Annual Meeting 2015 and

Pre-meeting Course: Arthritis

July $15-18,2015$

Long Beach, California, USA

www.aofas.org/annualmeeting

36th SICOT Orthopaedic World Congress

September 17-19, 2015

Guangzhou, China

http://www.sicot.org/guangzhou 


\section{Annual Conference of Indian Foot and Ankle Society}

American Orthopaedic Foot \& Ankle Society Surgical Complications of the

Foot and Ankle Course

November 5-7, 2015

Tampa, Florida, USA

www.aofas.org

\section{European Foot and Ankle Society Advanced Symposium}

December 4-5, 2015

Genoa, Italy

efas@eventplus.ie

\section{Indian Orthopaedic Association Congress IOACON 2015}

December 9-14, 2015

Jaipur, India

http://ioacon2015.org/

\section{4th European Foot and Ankle Society Cadaver Course}

February, 2016

Barcelona, Spain

efas@eventplus.ie

\section{8th EFAS Instructional Course}

April 2016, location TBC

efas@eventplus.ie

Foot International 2016, European Foot and Ankle Society, DAF, i-FAB Congress

June 23-25, 2016

Berlin, Germany

efas@eventplus.ie

6th Asian Federation of Foot and Ankle Surgeons (AFFAS) Meeting

November 19-20, 2016

Nara, Japan

affas@c-linkage.co.jp

\section{8th Annual Conference of Indian Foot and Ankle Society}

\section{Renowned National Faculty}

- For Registration kindly send you personal details and Demand Draft/Cheque in favour of 'IFASCON 2015' payable at Ludhiana and should be sent to the Conference Secretariat.

- For PG students, a certificate from head of department/institution is compulsory.

- For Spot registrations only cash will be accepted. Spot registrations will be provided delegate kit subject to availability.

- Last data for Abstract Submission is 31st May 2015. 